\title{
SHP2 expression is an independent negative prognostic factor in human breast cancer
}

\author{
S Muenst ${ }^{1,2}$, EC Obermann ${ }^{1}$, F Gao $^{3}$, D Oertli ${ }^{4}$, CT Viehl ${ }^{4}$, WP Weber ${ }^{4}$, T Fleming $^{2}$, WE \\ Gillanders $^{2}$, and SD Soysal ${ }^{2,4}$ \\ ${ }^{1}$ Institute of Pathology, University Hospital Basel, Switzerland ${ }^{2}$ Department of Surgery, \\ Washington University School of Medicine, St. Louis, USA ${ }^{3}$ Division of Biostatistics, Washington \\ University School of Medicine, St. Louis, USA ${ }^{4}$ Department of Surgery, University Hospital Basel, \\ Switzerland
}

\begin{abstract}
Aims-Src-homology-phosphotyrosyl-phosphatase 2 (SHP2) is a ubiquitously expressed phosphatase that plays an essential role in the downstream signaling pathways of multiple growth factor receptors, thus representing a potential target for cancer therapy. Recent studies suggest that SHP2 contributes to tumor initiation, progression and metastasis in breast cancer, yet the impact of SHP2 expression on prognosis in human breast cancer has not been evaluated.

Methods and Results-To further explore the role of SHP2 in breast cancer, we conducted an immunohistochemical study on a tissue microarray encompassing 1401 formalin-fixed breast cancer specimens with detailed clinical annotation and outcomes data. 651 of 1401 (46\%) evaluable breast cancers were positive for SHP2. SHP2 expression was positively associated with tumor grade, lymph node status, and tumor stage. In univariate survival analysis, cases with SHP2 expression had a significantly worse overall survival (OS). In multivariate analysis, SHP2 remained an independent negative prognostic factor for OS. SHP2 expression was a negative prognostic factor for OS in the luminal A and the luminal B HER2- intrinsic subtypes.
\end{abstract}

Conclusions-Our data demonstrate for the first time that SHP2 is an independent predictor of survival in breast cancer, suggesting that SHP2 may be a potential target for therapy.

\section{Keywords}

SHP2; PTPN11; breast cancer; prognostic factor

\section{INTRODUCTION}

Protein tyrosine phosphatases (PTP) play a major role in a broad range of cellular functions, including proliferation, differentiation, intercellular communication and motility 1,2 .

Corresponding author: Simone Muenst, MD, Institute of Pathology, University Hospital Basel, Schönbeinstrasse 40, CH-4032 Basel, Switzerland, Phone +41 6126527 57, Fax +41 6126531 94, muensts@uhbs.ch. 
Multiple studies indicate that PTPs function in collaboration with protein tyrosine kinases in regulating the levels of cellular tyrosine phosphorylation, thereby modulating signaling pathways in diverse physiological processes ${ }^{1,2}$. Src homology phosphotyrosyl phosphatase 2 (SHP2) is a ubiquitously expressed cytoplasmic enzyme that plays an important role in the downstream signaling pathways of multiple growth factor receptors such as the epidermal growth factor receptor (EGFR) and the platelet derived growth factor receptor (PDGFR) ${ }^{3}$.

Although it is not completely understood how SHP2 modulates these signaling cascades, activation of growth factor receptors, including EGFR, results in SHP2 phosphorylation, and extracellular signal regulated protein kinase (ERK) activation ${ }^{4}$. ERK in turn phosphorylates different substrates in the cytosol and traverses the nuclear membrane to activate transcription factors 5 .

There is accumulating evidence suggesting that SHP2 may play a crucial role in cancer. SHP2 activation can occur either downstream of oncoproteins or by mutations ${ }^{6}$. Germline mutations in the PTPN11 gene which encodes SHP2 are reported in about half of the patients with Noonan Syndrome, an autosomal dominant disorder whose features include an increased risk of myeloproliferative disorders ${ }^{7}$. Somatic gain-of-function mutations of the PTPN11 gene have also been identified in patients with juvenile myeloid leukemia as well as in some cases of lung adenocarcinomas, colon cancers, thyroid cancers, melanomas and neuroblastomas ${ }^{7-9}$. These genetic observations identified PTPN11 as the first oncogene in the PTP superfamily ${ }^{1}$. Activation of the Ras-ERK and the PI3K-AKT signaling pathways are thought to be the principal transforming effect of mutant SHP2 ${ }^{3}, 10$. Since wildtype SHP2 modulates important signaling events downstream of various growth factor receptors and proto-oncongenes, PTPNII is now considered a bona fide oncogene ${ }^{7}$ and thus represents a potential target for cancer therapy ${ }^{10}$.

Of note, there is also evidence that SHP2 may act as a tumor suppressor in liver cancer ${ }^{11}$. Deletion of PTPN11 in hepatocytes leads to development of hepatocellular tumors in aged mice, and SHP2 ablation increases development of diethylenenitrite-induced hepatocellular carcinoma in a mouse model ${ }^{11}$.

Several studies to date suggest a role for SHP2 in breast cancer. An immunohistochemistry study by Zhou et al. ${ }^{3}$ reported overexpression of SHP2 in $72 \%$ of invasive ductal breast cancers and an association between SHP2 expression, tumor grade, and lymph node metastasis. The authors demonstrated that the immunohistochemical method they used was able to discriminate between SHP2 overexpression in breast cancer compared to normal breast tissue, suggesting that immunohistochemistry is a robust technique for evaluating SHP2 expression ${ }^{3}$. SHP2 expression was also reported to be associated with both Human Epidermal Growth Factor Receptor 2 (HER2) expression, as well as with estrogen receptor (ER) expression and progesterone receptor (PR) expression ${ }^{3}$. Consistent with these findings are two studies suggesting that SHP2 plays a role in HER2-induced breast cancer signaling/ transformation $3,12,13$. In the first study, inhibition of SHP2 in HER2-positive breast cancer cell lines abrogated survival signaling, resulting in a conversion to a normal breast epithelial phenotype ${ }^{13}$. This study also showed that SHP2 promotes epithelial-mesenchymal transition in breast cancer cell lines with downregulation of E-cadherin and upregulaton of 
fibronectin and vimentin ${ }^{13}$. In the second study, the authors showed that HER2 is a direct substrate for SHP2 and that dephosphorylation of a single autophosphorylsation site in HER 2 by SHP2 leads to enhanced HER 2 signaling and cell transformation ${ }^{12}$. To date no mutations or amplifications of PTPN11 in breast cancer cell lines have been identified. However, the gene encoding the SHP2-activating protein growth factor receptor bound 2associated protein 2 (GAB2) is amplified and overexpressed in 10-15\% of human breast cancers 14,15 .

Recently, Aceto et al. confirmed an important role for SHP2 in tumor initiation, progression and metastasis in HER2 ${ }^{+}$and triple-negative breast cancers ${ }^{6}$. The authors demonstrated that specific ablation of SHP2 led to reduced breast cancer invasion in vitro, as well as decreased tumor growth and lung metastases in a mouse model. They also found a high expression of SHP2 protein by immunohistochemistry in 91\% of breast cancers, but SHP2 expression did not correlate with histological subtype or clinicopathological parameters. Moreover, they identified a set of "SHP2 signature genes" that are activated in the presence of SHP2 signaling in a subset of human breast cancers and are associated with invasive behavior and poor prognosis 6 .

Despite this interest in defining the role of SHP2 in cancer biology at the molecular level, the impact of SHP2 expression on prognosis in human breast cancer has not been studied. To address this issue and to further explore the role of SHP2 in human breast cancer, we performed immunohistochemistry studies on a breast cancer tissue microarray (TMA) encompassing a total of 1401 formalin fixed breast cancer specimens with detailed clinical annotation and outcomes data.

\section{MATERIALS AND METHODS}

\section{Tissue microarray}

We used a tissue micoroarray (TMA) encompassing 2020 breast cancer tissue punches from 1579 formalin-fixed and paraffin-embedded tumor samples, which were collected from patients diagnosed with primary breast cancer between 1985 and 2007 at the Institute for Pathology, University of Basel and the Viollier Institute in Basel, Switzerland. The tissue samples were brought into a TMA format as previously described ${ }^{16}$. In brief, $0.6 \mathrm{~mm}$ tissue cylinders were punched out of donor tumor tissue blocks and transferred into a recipient paraffin block using a semiautomated tissue arrayer. Each TMA contained a number of tumor punches ranging from 159 to 522 . Histopathological data was obtained from the pathology reports, and raw patients survival data was obtained from the Cancer Registry of Basel or from the patient's attending physicians. Retrieval of tissue and clinical data was performed according to the regulations of the local institutional review boards and data safety laws with specific regard to ethical standards and patient confidentiality.

\section{Immunohistochemistry}

SHP2 immunohistochemistry was performed using standard techniques. Briefly, $4 \mu \mathrm{m}$ sections of the TMA blocks were incubated overnight with primary anti-SHP2 antibody (LifeSpan Biosciences, Inc., Seattle, WA, Catalog \# LS-B542) after heat induced antigen 
retrieval with Citrate buffer at pH 6. Standard DAB technique (Dako EnVision ${ }^{+}$System; labelled polymer anti-rabbit followed by Liquid $\mathrm{DAB}^{+}$substrate chromogen system) was employed for immunostaining. Counterstaining was performed with Harris hematoxylin solution.

Consistent with the known intracellular localization of SHP2, breast cancers with positive SHP2 staining showed a cytoplasmic distribution of SHP2. Using the same scoring system as described by Zhou et al. ${ }^{3}$, strong cytoplasmatic reactivity of $>10 \%$ of all tumor cells was designated as $3+$, moderate reactivity as $2+$, and faint reactivity as $1+$ (Figure 1 ). Faint reactivity not discernible above background or absence of any staining was considered negative for SHP2 expression. A reactivity of $2+$ and $3+$ was considered positive for SHP2 expression. Microscopically normal portions of breast specimens were used as controls.

The staining intensity of ER, PR, and HER2 was scored as described previously ${ }^{17}$. In brief, tumors were considered positive for ER if they showed nuclear staining in more than $10 \%$ of tumor cells, with an intensity score between 1 and 3 .

\section{Statistical analysis}

The primary outcome was overall survival (OS), which was defined as the time from the first operation to death due to any cause. Survivors were censored at the date of last contact. The distributions of patient and clinical characteristics between SHP2-positive and negative tumors were compared using Chi-square test, Wilcoxon rank sum test, or two-sample t-test as appropriate. Survival curves by SHP2 status were estimated using the Kaplan-Meier product-limit method and compared by log-rank test. Univariate Cox proportional hazard models were fit to identify factors significantly related to overall survival. To assess whether SHP2 was an independent predictor of survival, a multivariate Cox model was constructed to adjust other patient/clinical characteristics that were significant in the univariate analyses. Two-way interaction terms between SHP2 and other factors in the multivariate Cox model were also assessed. All analyses were two-sided and significance was set at a $p$ value of 0.05. Statistical analyses were performed using SAS (SAS Institutes, Cary, NC).

\section{RESULTS}

A total of 1401 cases were evaluable for this study. The mean follow up time was 80.8 months (range 1 to 263 months), and the mean age of the patients was 63 years (range 27 to 101 years). Demographic information for the patients included in this study can be found in Table 1.

Using the afore mentioned scoring system, a total of 651 of 1401 (46\%) evaluable primary breast cancers were positive for SHP2.

SHP2 expression showed a positive correlation with AJCC primary tumor classification, lymph node status, and tumor grade (Table 2). SHP2 expression also varied significantly across the different histologic types of breast cancer, being highest in the invasive ductal cancers $(47.3 \%)$ and lowest in cribriform cancers $(31.7 \%, p=0.0250)$ (Table 3$)$. 
Breast cancer intrinsic subtypes were originally defined by gene expression profiling ${ }^{18}$ but can be approximated using immunohistochemistry for ER, PR, Ki-67 and HER2 ${ }^{12}$. Breast cancer intrinsic subtypes are known to have distinct epidemiological risk factors, and vary in terms of prognosis, and response to therapy ${ }^{12}$. There was no significant difference in SHP2 expression across the different breast cancer intrinsic subtypes ( $p=0.9108$, Table 4$)$, as defined by the St. Gallen consensus conference ${ }^{19}$.

SHP2 showed a positive correlation with expression of the protein tyrosine phosphatase 1B (PTP1B) ( $p=0.026)$ (data not shown). However, no significant association was found with ER or PR receptor status (data not shown), or HER2 status (Table 2).

Univariate survival analyses showed that breast cancers with positive SHP2 expression had a significantly worse overall survival (OS) (HR $=1.458, p<0.0001)$. In the different breast cancer intrinsic subtypes, SHP2 expression proved to be a negative prognostic factor for OS in univariate analysis in the luminal A (HR $=1.813, p=0.0323)$ and the luminal B HER2subtypes (HR $=1.572, p=0.0005)$. No difference in OS with SHP2 expression was found in the Luminal B HER2 ${ }^{+}$subtype $(\mathrm{HR}=1.346, p=0.237)$, HER2 subtype $(\mathrm{HR}=1.457, p=$ $0.1765)$, or basal-like subtype $(\mathrm{HR}=1.076, p=0.7024)$ (Figure 2 and Table 5).

In multivariate analysis, after adjusting for age, grade, tumor stage, lymph node status, and intrinsic subtype, SHP2 remained an independent negative prognostic factor for OS (HR = $1.239, p=0.0177)$. The 2-way interaction term between SHP2 and intrinsic subtype was not significant $(p=0.91)$ (Table 6).

\section{DISCUSSION}

We examined the expression of SHP2 in a large cohort of 1401 primary breast cancers and demonstrate for the first time that expression of SHP2 is an independent negative prognostic factor for overall survival in human breast cancer. Our study has important implications as small molecule inhibitors targeting SHP2 are currently under development.

In our study, SHP2 expression in human breast cancer was lower than previously reported. We observed SHP2 expression in 651 of 1401 cases (46\%), compared to $72 \%$ in the study by Zhou et al. ${ }^{3}$, and $91 \%$ in the study by Aceto et al. ${ }^{6}$. Of note, all three studies used the same scoring system, but different antibodies. The antibody we used is directed against the N-Terminus of SHP2 and has been validated for immunohistochemistry. Of note, we also stained normal breast tissue, which showed faint epithelial staining that was barely discernible beyond background (data not shown). This provides additional evidence that the antibody we used is suitable for the detection of SHP2 expression in breast cancer.

Consistent with Zhou et al., we found a positive association between SHP2 expression, tumor grade, and lymph node metastasis in breast cancer, although to a lesser extent ${ }^{3}$. Several studies have demonstrated that SHP2 promotes cell migration and invasion ${ }^{6,22}$. Consistent with these findings, SHP2 expression was highly associated with lymph node metastasis in our study $(\mathrm{p}=0.0263)$. 
In contrast with Zhou et al. ${ }^{3}$, there was no association between SHP2 expression and ER or PR expression, and SHP2 expression was not significantly different across the different intrinsic subtypes, underscoring the fact that SHP2 represents a broadly expressed protein in human breast cancer and impacts breast cancer prognosis independently.

Although it is known that SHP2 is an important downstream effector in EGFR family signaling ${ }^{23}$ and a study by Zhou et al. showed that SHP2 directly dephosphorylates HER2 and leads to enhanced HER2 signaling ${ }^{12}$, we found no correlation between the SHP2 expression and HER2 amplification in this cohort. This suggests that the impact of SHP2 on human breast cancer is not dependent on HER2. We also observed a positive association between SHP2 expression and protein tyrosine phosphatase 1B (PTP1B) expression. A study by Ferrari et al. reports that SHP2 is a substrate of PTP1B, and SHP2 expression is specifically modulated by PTP1B ${ }^{24}$. This may explain the association between these two protein phosphatases in our study.

Of note, SHP2 expression proved to be an independent negative prognostic factor for OS in breast cancer. To our knowledge this is a new finding as no reports have assessed the impact of SHP2 expression on prognosis in breast cancer. Multiple studies have shown that SHP2 plays an important role in cellular transformation and cell signaling ${ }^{20,21}$, Since no mutations of PTPN11 have been identified in breast cancer to date ${ }^{625}$, it is likely that overexpression of SHP2 occurs downstream of other mutated proto-oncogenes ${ }^{6}$ such as EGFR and PDGFRa ${ }^{7}$. Investigating the specific role of SHP2 in breast cancer, Aceto et al. have shown that SHP2 acts predominantly by activating the extracellular signal-regulated kinases ERK1 and ERK2 ${ }^{6}$, which in turn leads to altered transcription of cell cycle genes and uncontrolled cell growth.

Additionally, the gene encoding the SHP2 binding protein GAB2 is frequently amplified in breast cancers 6,7 and can, by acting through SHP2, lead to excessive proliferation of mammary epithelial cells as well as potentiate the effect of HER2 dependent cell transformation and carcinogenesis ${ }^{9}$. It is thus very feasible that SHP2 expression promotes oncogenic signaling and dedifferentiation in breast cancer, contributing to a worse prognosis. Our results are consistent with the findings by Aceto et al., demonstrating that SHP2 promotes breast cancer progression and metastasis in murine models ${ }^{6}$ and thus provide an important link between studies in breast cancer cell lines and human breast cancer.

In subgroup analyses SHP2 expression was associated with worse OS in two of the intrinsic breast cancer subtypes (luminal A and the luminal B HER2- ${ }^{-}$subtype). We also observed a trend towards worse OS with SHP2 expression in two other subtypes (luminal B HER2 ${ }^{+}$and HER2 subtype). There were significantly more cases in the luminal A and the luminal B HER2- ${ }^{-}$subtypes, so the lack of statistical significance in the luminal B HER2 ${ }^{+}$and HER2 subtypes may be based on lack of statistical power. The basal-like subtype was the only subtype where SHP2 expression did not appear to have any effect on OS. This is significant since the study by Aceto et al. showed a fundamental role for SHP2 in triple negative breast cancers ${ }^{6}$. Their work however, is based on a limited number of human breast cancer cell 
lines grown in mice and these cell lines may not capture the complex biology and heterogeneity of triple negative human breast cancer.

Our study is the largest TMA study to date to evaluate SHP2 expression in breast cancer, and is the first to demonstrate that SHP2 expression is an independent predictor of prognosis in breast cancer. Our data suggests that SHP2 represents a potential target in human breast cancer. This has important implications as selective small molecule inhibitors of SHP2 are currently under development ${ }^{23}, 26$. Routine staining for SHP2 in breast cancer could be used to identify patients eligible for targeted therapy, but could also be used to identify those patients with a more aggressive course of disease. Additional studies are needed to define the role of SHP2 in human breast cancer.

\section{Acknowledgments}

The authors wish to acknowledge the support of the Biostatistics Core, Siteman Comprehensive Cancer Center WUSM and NCI Cancer Center Support Grant P30 CA091842.

\section{REFERENCES}

1. Jiang ZX, Zhang ZY. Targeting ptps with small molecule inhibitors in cancer treatment. Cancer Metastasis Rev. 2008; 27:263-272. [PubMed: 18259840]

2. Hatakeyama M. Oncogenic mechanisms of the helicobacter pylori caga protein. Nat Rev Cancer. 2004; 4:688-694. [PubMed: 15343275]

3. Zhou X, Coad J, Ducatman B, Agazie YM. Shp2 is up-regulated in breast cancer cells and in infiltrating ductal carcinoma of the breast, implying its involvement in breast oncogenesis. Histopathology. 2008; 53:389-402. [PubMed: 18643929]

4. Furcht CM, Munoz Rojas AR, Nihalani D, Lazzara MJ. Diminished functional role and altered localization of shp2 in non-small cell lung cancer cells with egfr-activating mutations. Oncogene. 2012

5. Quintanar-Audelo M, Yusoff P, Sinniah S, Chandramouli S, Guy GR. Sprouty-related ena/ vasodilator-stimulated phosphoprotein homology 1-domain-containing protein (spred1), a tyrosineprotein phosphatase non-receptor type $11(\mathrm{shp} 2)$ substrate in the ras/extracellular signal-regulated kinase (erk) pathway. J Biol Chem. 2011; 286:23102-23112. [PubMed: 21531714]

6. Aceto N, Sausgruber N, Brinkhaus H, et al. Tyrosine phosphatase shp2 promotes breast cancer progression and maintains tumor-initiating cells via activation of key transcription factors and a positive feedback signaling loop. Nat Med. 2012

7. Chan G, Kalaitzidis D, Neel BG. The tyrosine phosphatase shp2 (ptpn11) in cancer. Cancer Metastasis Rev. 2008; 27:179-192. [PubMed: 18286234]

8. Tartaglia M, Niemeyer CM, Fragale A, et al. Somatic mutations in ptpn11 in juvenile myelomonocytic leukemia, myelodysplastic syndromes and acute myeloid leukemia. Nat Genet. 2003; 34:148-150. [PubMed: 12717436]

9. Mohi MG, Neel BG. The role of shp2 (ptpn11) in cancer. Curr Opin Genet Dev. 2007; 17:23-30. [PubMed: 17227708]

10. Ostman A, Hellberg C, Bohmer FD. Protein-tyrosine phosphatases and cancer. Nat Rev Cancer. 2006; 6:307-320. [PubMed: 16557282]

11. Bard-Chapeau EA, Li S, Ding J, et al. Ptpn11/shp2 acts as a tumor suppressor in hepatocellular carcinogenesis. Cancer Cell. 2011; 19:629-639. [PubMed: 21575863]

12. Zhou X, Agazie YM. Molecular mechanism for shp2 in promoting her2-induced signaling and transformation. J Biol Chem. 2009; 284:12226-12234. [PubMed: 19261604]

13. Zhou XD, Agazie YM. Inhibition of shp2 leads to mesenchymal to epithelial transition in breast cancer cells. Cell Death Differ. 2008; 15:988-996. [PubMed: 18421299] 
14. Bentires-Alj M, Gil SG, Chan R, et al. A role for the scaffolding adapter gab2 in breast cancer. Nat Med. 2006; 12:114-121. [PubMed: 16369543]

15. Bocanegra M, Bergamaschi A, Kim YH, et al. Focal amplification and oncogene dependency of gab2 in breast cancer. Oncogene. 2010; 29:774-779. [PubMed: 19881546]

16. Bubendorf L, Nocito A, Moch H, Sauter G. Tissue microarray (tma) technology: Miniaturized pathology archives for high-throughput in situ studies. J Pathol. 2001; 195:72-79. [PubMed: 11568893]

17. Tapia C, Schraml P, Simon R, et al. Her2 analysis in breast cancer: Reduced immunoreactivity in fish non-informative cancer biopsies. Int J Oncol. 2004; 25:1551-1557. [PubMed: 15547690]

18. Perou CM, Sorlie T, Eisen MB, et al. Molecular portraits of human breast tumours. Nature. 2000; 406:747-752. [PubMed: 10963602]

19. Goldhirsch A, Wood WC, Coates AS, Gelber RD, Thurlimann B, Senn HJ. Strategies for subtypes--dealing with the diversity of breast cancer: Highlights of the st. Gallen international expert consensus on the primary therapy of early breast cancer 2011. Ann Oncol. 2011; 22:17361747. [PubMed: 21709140]

20. Agazie YM, Movilla N, Ischenko I, Hayman MJ. The phosphotyrosine phosphatase shp2 is a critical mediator of transformation induced by the oncogenic fibroblast growth factor receptor 3 . Oncogene. 2003; 22:6909-6918. [PubMed: 14534538]

21. Ralston A, Rossant J. How signaling promotes stem cell survival: Trophoblast stem cells and shp2. Dev Cell. 2006; 10:275-276. [PubMed: 16516829]

22. Yu DH, Qu CK, Henegariu O, Lu X, Feng GS. Protein-tyrosine phosphatase shp-2 regulates cell spreading, migration, and focal adhesion. J Biol Chem. 1998; 273:21125-21131. [PubMed: 9694867]

23. Agazie YM, Hayman MJ. Molecular mechanism for a role of shp2 in epidermal growth factor receptor signaling. Mol Cell Biol. 2003; 23:7875-7886. [PubMed: 14560030]

24. Ferrari E, Tinti M, Costa S, et al. Identification of new substrates of the protein-tyrosine phosphatase ptp1b by bayesian integration of proteome evidence. J Biol Chem. 2011; 286:41734185. [PubMed: 21123182]

25. Bentires-Alj M, Paez JG, David FS, et al. Activating mutations of the noonan syndrome-associated shp2/ptpn11 gene in human solid tumors and adult acute myelogenous leukemia. Cancer Res. 2004; 64:8816-8820. [PubMed: 15604238]

26. Yu ZH, Chen L, Wu L, Liu S, Wang L, Zhang ZY. Small molecule inhibitors of shp2 tyrosine phosphatase discovered by virtual screening. Bioorg Med Chem Lett. 2011; 21:4238-4242. [PubMed: 21669525] 


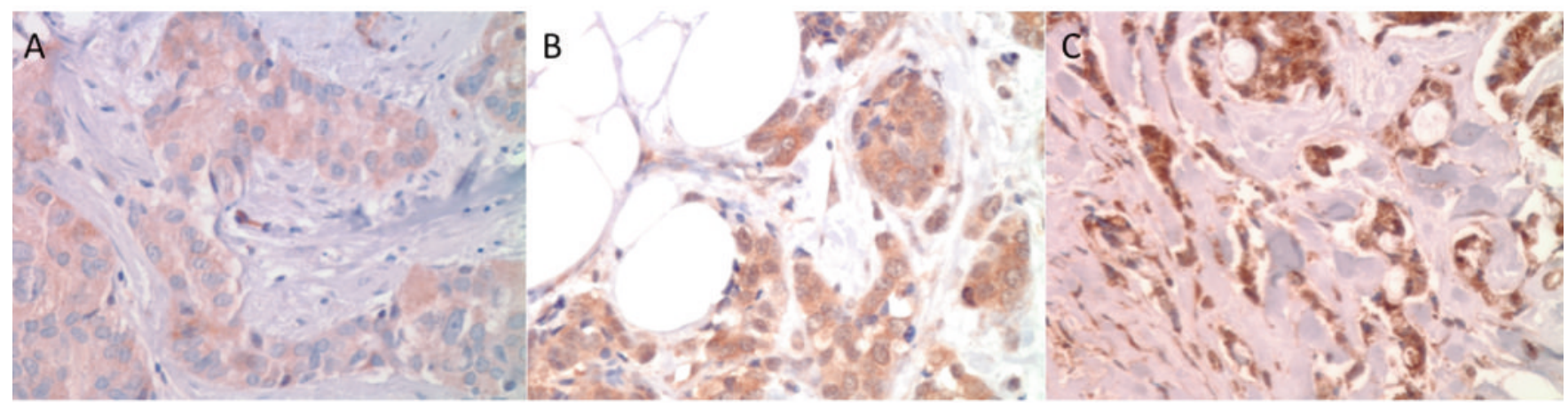

Figure 1.

Representative photographs of SHP2 expression in breast cancer with a weak cytoplasmic expression (1+) b moderate cytoplasmic expression (2+) c strong cytoplasmic expression $(3+)$ of tumor cells. SHP2 staining, magnification 400x. 
A). All

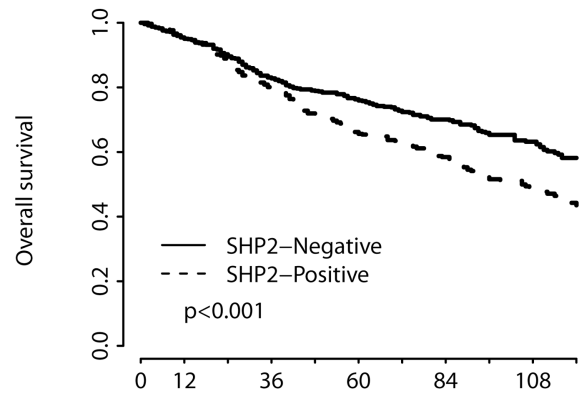

Time to death (months)

C). Lum B, Her2-

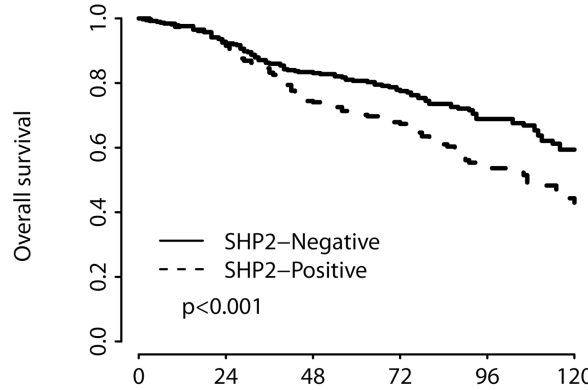

Time to death (months)

E). Her2+

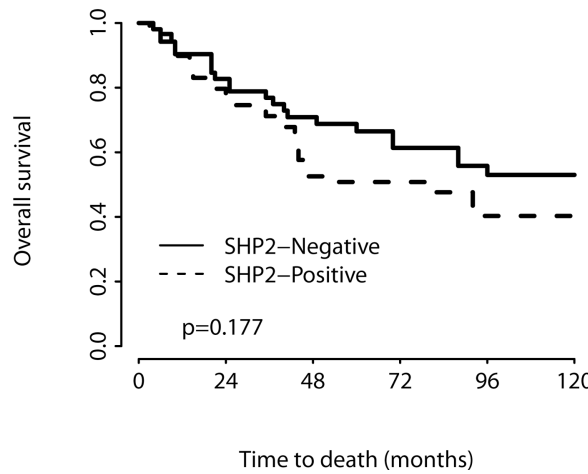

B). Lum A

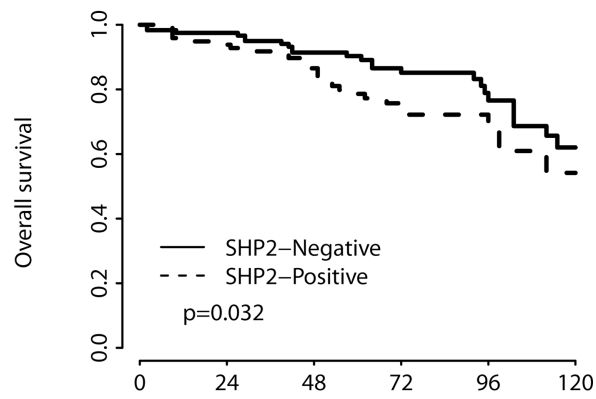

Time to death (months)

D). Lum B, Her2+

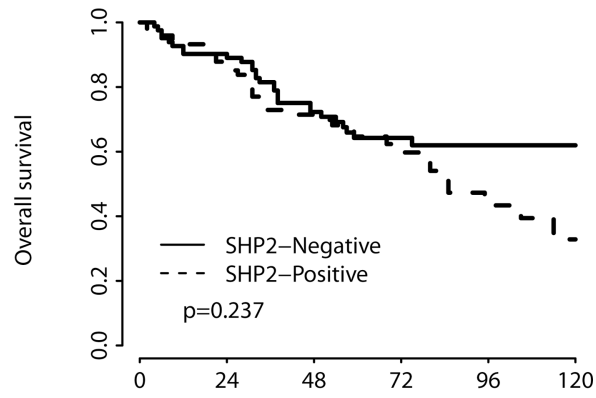

Time to death (months)

F). Basal-like

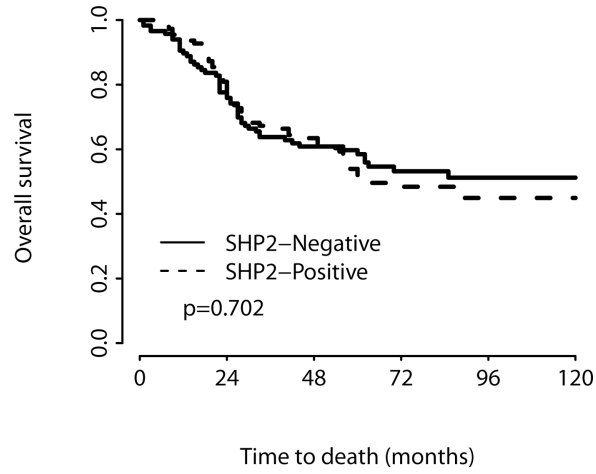

Figure 2.

Kaplan-Meier survival curve for a overall survival depending on SHP2 expression (univariate analysis) b-f Kaplan-Meier survival curve for overall survival depending on SHP2 expression for the intrinsic breast cancer subtypes. 
Table 1

Basic demographic data for 1401 evaluable breast cancer cases

\begin{tabular}{|c|c|c|}
\hline Mean tumor size (mm) & \multicolumn{2}{|c|}{30.7} \\
\hline Mean age at diagnosis (years) & \multicolumn{2}{|c|}{63.5} \\
\hline Tumor size & Number (n) & Percent $(\%)$ \\
\hline pT1 & 385 & 27.5 \\
\hline pT2 & 737 & 52.6 \\
\hline pT3 & 107 & 7.6 \\
\hline pT4 & 172 & 12.3 \\
\hline \multicolumn{3}{|l|}{ Lymph node involvement } \\
\hline pNO & 725 & 51.8 \\
\hline $\mathrm{pN} 1$ & 539 & 38.6 \\
\hline $\mathrm{pN} 2$ & 134 & 9.6 \\
\hline \multicolumn{3}{|l|}{ Tumor grade } \\
\hline 1 & 323 & 23.0 \\
\hline 2 & 576 & 41.1 \\
\hline 3 & 502 & 35.9 \\
\hline \multicolumn{3}{|l|}{ Histologic subtype } \\
\hline Invasive ductal & 992 & 70.9 \\
\hline Invasive lobular & 203 & 14.5 \\
\hline Mucinous & 38 & 2.7 \\
\hline Apocrine & 17 & 1.2 \\
\hline Cribriform & 41 & 2.9 \\
\hline Papillary & 19 & 1.4 \\
\hline Medullary & 45 & 3.2 \\
\hline Other & 44 & 3.2 \\
\hline \multicolumn{3}{|l|}{ Intrinsic subtype } \\
\hline $\begin{array}{c}\text { Luminal A } \\
\left(\mathrm{ER}^{+} \text {and/or } \mathrm{PR}^{+}, \mathrm{HER}^{-}, \mathrm{Ki}-67<14 \%\right)\end{array}$ & 217 & 15.6 \\
\hline $\begin{array}{c}\text { Luminal B (HER2-negative) } \\
\left.\mathrm{ER}^{+} \text {and/or } \mathrm{PR}^{+}, \mathrm{HER}^{-}, \mathrm{Ki}-67 \geq 14 \%\right)\end{array}$ & 683 & 49.0 \\
\hline $\begin{array}{l}\text { Luminal B (HER2-positive) } \\
\left(\mathrm{ER}^{+} \text {and/or } \mathrm{PR}^{+}, \mathrm{HER} 2^{+}\right)\end{array}$ & 156 & 11.2 \\
\hline HER2 type $\left(\mathrm{ER}^{-}\right.$or $\left.\mathrm{PR}^{-}, \mathrm{HER}^{+}\right)$ & 111 & 7.9 \\
\hline Basal-like (ER $\left.{ }^{-}, \mathrm{PR}^{-}, \mathrm{HER} 2^{-}\right)$ & 227 & 16.3 \\
\hline
\end{tabular}




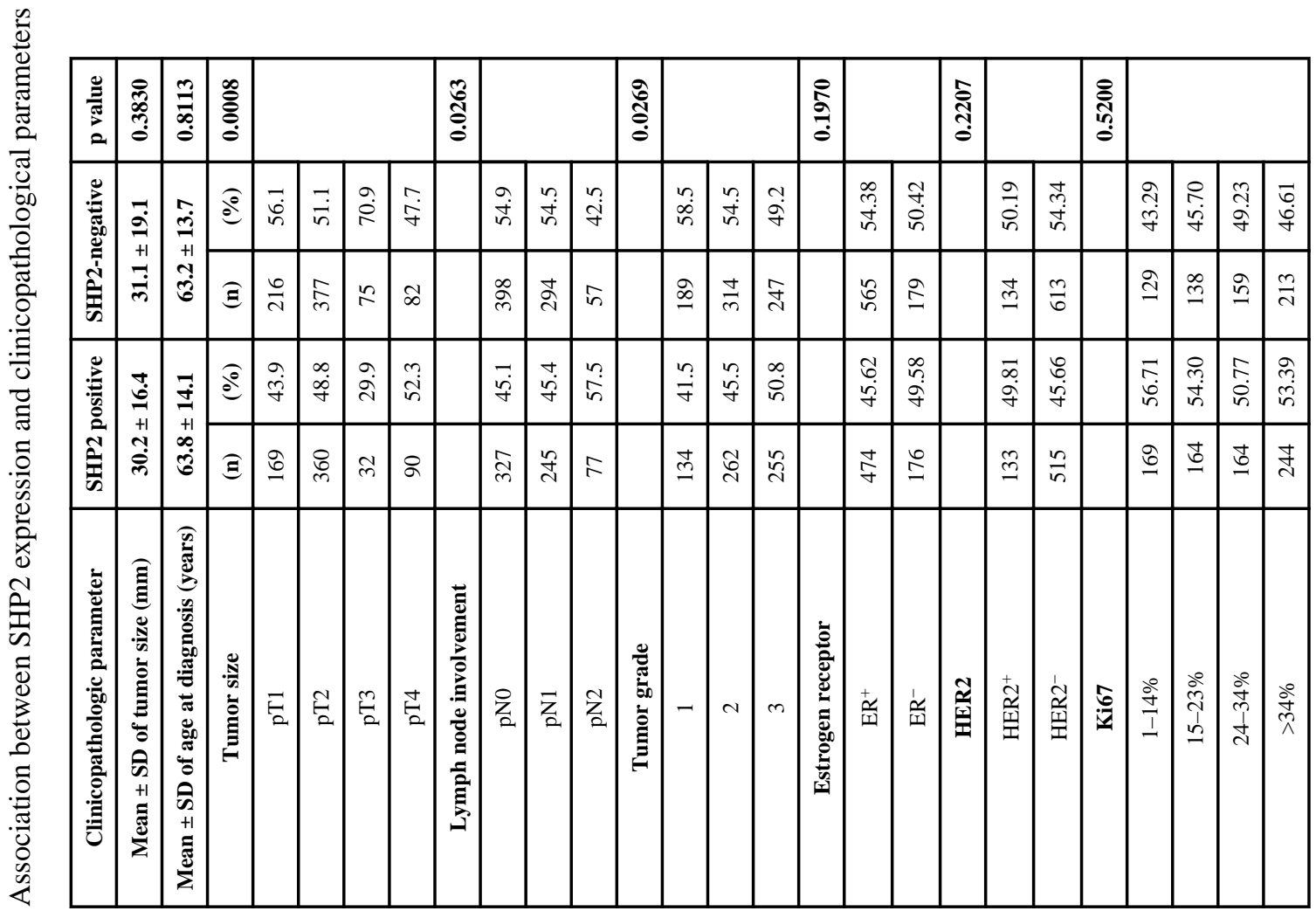




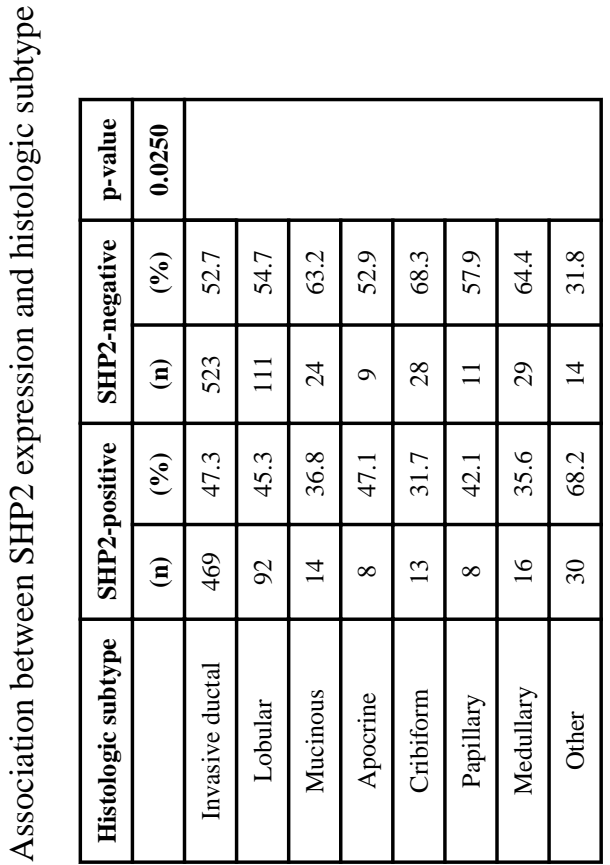

Histopathology. Author manuscript; available in PMC 2014 July 16. 


\begin{tabular}{|c|c|c|c|c|c|c|}
\hline 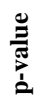 & $\mid \begin{array}{l}\infty \\
\stackrel{0}{0} \\
\stackrel{0}{0}\end{array}$ & & & & & \\
\hline 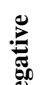 & 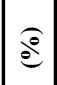 & $\begin{array}{l}\text { 伃 } \\
\text { in }\end{array}$ & $\vec{n}$ & $\begin{array}{l}0 \\
i \\
i\end{array}$ & $\begin{array}{l}\infty \\
\dot{f} \\
\dot{f}\end{array}$ & $\frac{n}{n}$ \\
\hline 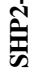 & $\widehat{\Xi}$ & $\stackrel{\partial}{\exists}$ & $\stackrel{\circ}{\infty}$ & 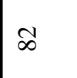 & $\approx$ & $\Xi$ \\
\hline 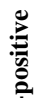 & 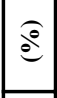 & 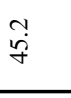 & $\stackrel{\partial}{\dot{f}}$ & $\begin{array}{l}\vec{\partial} \\
\vec{f}\end{array}$ & $\vec{n}$ & 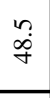 \\
\hline$\tilde{\tilde{E}}$ & $\widehat{\Xi}$ & $\stackrel{\circ}{\circ}$ & 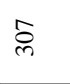 & $\stackrel{t}{亡}$ & in & $\stackrel{\circ}{\varrho}$ \\
\hline 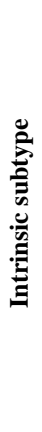 & & 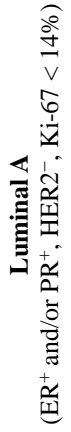 & 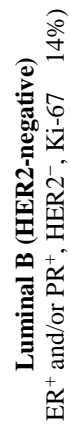 & 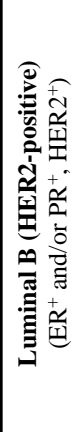 & 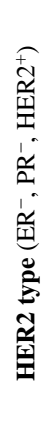 & 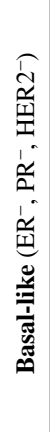 \\
\hline
\end{tabular}

Histopathology. Author manuscript; available in PMC 2014 July 16. 
Table 5

Univariate analyses for all cases, and by intrinsic subtype, for the effect of SHP2 expression on overall survival

\begin{tabular}{|c|c|c|}
\hline SHP2 expression, all cases & Hazard Ratio $(\mathbf{9 5 \%}$ CI) & p-value \\
\hline SHP2-positive & $1.458(1.227-1.733)$ & $<.0 .0001$ \\
\hline SHP2 expression, by intrinsic subtype & & \\
\hline Luminal A & $1.813(1.052-3.127)$ & $\mathbf{0 . 0 3 2 3}$ \\
\hline${\text { Luminal B }\left(H E R 2^{-}\right)}^{-}$ & $1.572(1.218-2.030)$ & $\mathbf{0 . 0 0 0 5}$ \\
\hline Luminal B $\left(H E R 2^{+}\right)$ & $1.346(0.822-2.204)$ & $\mathbf{0 . 2 3 7 0}$ \\
\hline HER2 type & $1.457(0.844-2.513)$ & $\mathbf{0 . 1 7 6 5}$ \\
\hline Basal-like & $1.076(0.738-1.570)$ & $\mathbf{0 . 7 0 2 4}$ \\
\hline
\end{tabular}




\section{Table 6}

Multivariate analysisfor the effect of clinicopathologic parameters and SHP2expression on overall survival

\begin{tabular}{|c|c|c|}
\hline Clinicopathologic parameter & Hazard Ratio (95\% CI) & p-value \\
\hline Age(per 1-year ) & $1.037(1.030-1.044)$ & $<0.0001$ \\
\hline \multicolumn{3}{|l|}{ Tumor size } \\
\hline pT1 (reference) & 1 & \\
\hline pT2 & $1.619(1.247-2.101)$ & 0.0003 \\
\hline pT3 & $2.139(1.482-3.089)$ & $<0.0001$ \\
\hline pT4 & $2.269(1.639-3.143)$ & $<0.0001$ \\
\hline \multicolumn{3}{|l|}{ Lymph node involvement } \\
\hline pN1 (reference) & 1 & \\
\hline $\mathrm{pN} 1$ & $1.420(1.165-1.732)$ & 0.0005 \\
\hline $\mathrm{pN} 2$ & $2.602(1.969-3.440)$ & $<0.0001$ \\
\hline \multicolumn{3}{|l|}{ Tumor grade } \\
\hline BRE grade 1 (reference) & 1 & \\
\hline 2 & $1.582(1.196-2.092)$ & 0.0013 \\
\hline 3 & $2.527(1.889-3.381)$ & $<0.0001$ \\
\hline \multicolumn{3}{|l|}{ Intrinsic subtypes } \\
\hline Luminal A & 1 & \\
\hline Luminal B (HER2-) & $1.080(0.796-1.465)$ & 0.6205 \\
\hline Luminal B (HER2 $\left.2^{+}\right)$ & $1.268(0.870-1.848)$ & 0.2159 \\
\hline HER2 type & $1.169(0.774-1.764)$ & 0.4579 \\
\hline Basal-like & $1.941(1.369-2.752)$ & 0.0002 \\
\hline \multicolumn{3}{|l|}{ SHP2 expression, all cases* } \\
\hline & $1.239(1.038-1.480)$ & 0.0177 \\
\hline
\end{tabular}

Interaction between SHP2 expression and intrinsic subtypes not significant $(\mathrm{p}=0.91)$ 